Original Article

\title{
EXPLOITATION OF EDIBLE WILD PLANTS AS MEDICINAL PROPERTIES FOR PRIMARY HEALTH CARE SYSTEM IN BIDAR DISTRICT, NORTH KARNATAKA, INDIA
}

\author{
SUJATA M. P. ${ }^{*}$ \\ Department of Post Graduate Studies and Research in Botany, Gulbarga University Kalaburagi 585106, Karnataka, India \\ Email: sujaparma@gmail.com
}

Received: 21 Aug 2021, Revised and Accepted: 24 Dec 2021

\section{ABSTRACT}

Objective: Plants since times immemorial have been used practically in all societies as a source of medicine and food. The huge data of medicinal plants are available in the different geographical regions of India. Most of the knowledge and awareness about plants are found in the ethnic community and villages. The present paper tried to explore of medicinal plant property used in the study region with the help of ethnic communities.

Methods: A perspective documentation and cross-sectional studies of edible wild plants used as medicinal properties for various diseases of Bidar district. Surveys were undertaken in January 2018-July 2019in villages, ethnic people community and medicinal healers or local herbal practitioners in the study area.

Results: Total 42 medicinal healers' interviews were undertaken by the help of questioners or a standard survey datasheet. During the survey, a description of, 63 edible wild plant species belonging to 40 families and 53 genera was recorded. Total63 edible wild plants are used as medicine to cure different diseases by local medicinal practitioners of the district. Among 63 edible wild plants, the plant parts leaf (31 species) is most abundantly used, followed by fruits (22 species) and other plant parts are with less than 5 species i.e. 4, 3, 2 and single species.

Conclusion: However, day by day people will be attracted towards herbal medicine for the treatment of diseases. Many of the herbal plants have no side effect. So far, the present paper has been communicated to give more and more medicinal properties of edible wild plants.

Keywords: Medicinal plants, Edible wild plants, Diseases, Bidar district, North Karnataka

(C) 2022 The Authors. Published by Innovare Academic Sciences Pvt Ltd. This is an open-access article under the CC BY license (https://creativecommons.org/licenses/by/4.0/) DOI: https://dx.doi.org/10.22159/ijpps.2022v14i3.42928. Journal homepage: https://innovareacademics.in/journals/index.php/ijpps.

\section{INTRODUCTION}

Since many years' importance of, wild food plants have been used for food supplements during drought and famine. So, the consumption of such plants is called "wild food plants" or "famine foods" [1]. Plants, since times immemorial, have been used practically in all societies as a source of medicine and food. About 45,000 angiosperm plants have been used as food or nutritive value and medicinal value [2]. According to the world health organization (WHO), 1998 as many as 80\% of the world's population depend on traditional medicine for their primary healthcare. India is one of the mega-diversities of the world. Traditional systems of medicines are still very effective, particularly in rural areas of India for the treatment of various diseases $[3,4]$. Although the rate of medicinal plant utility is increasing day by day. But very little knowledge about medicinal plants is passing generation to generation used as medicine for health care. However, the present investigation includes the exploitation of edible wild plants with respect to medicine by tribals and rural people of the different socioeconomic communities of the district.

\section{MATERIALS AND METHODS}

\section{Study area}

Bidar district is situated in the North-Eastern part of Karnataka in India. The district is also called as crown of Karnataka state. The district is covering an area of $5448 \mathrm{sq}$. km., within $17^{\circ} 35^{\prime}$ and $18^{\circ} 25^{\prime}$ North latitudes and $76^{\circ} 42^{\prime}$ and $77^{\circ} 39^{\prime}$ East longitudes (fig. 1). The vegetation of forest division is classified into two sub-groups, namely, Southern tropical dry deciduous forests and Southern tropical thorn forests [5]. The district occupies various ethnic groups and socio-cultural communities. The main tribes are Lambani, Halakki and Kaadu kuruba scattered in both village and near forest areas of the district. Except these other tribes are Gond, Naikpod, Rajgond, Koli Dhor, Tokre Koli, Kolcha, Kolgha Koya, Bhine Koya, Rajkoya, Maha Malasar and Rathawa [6].

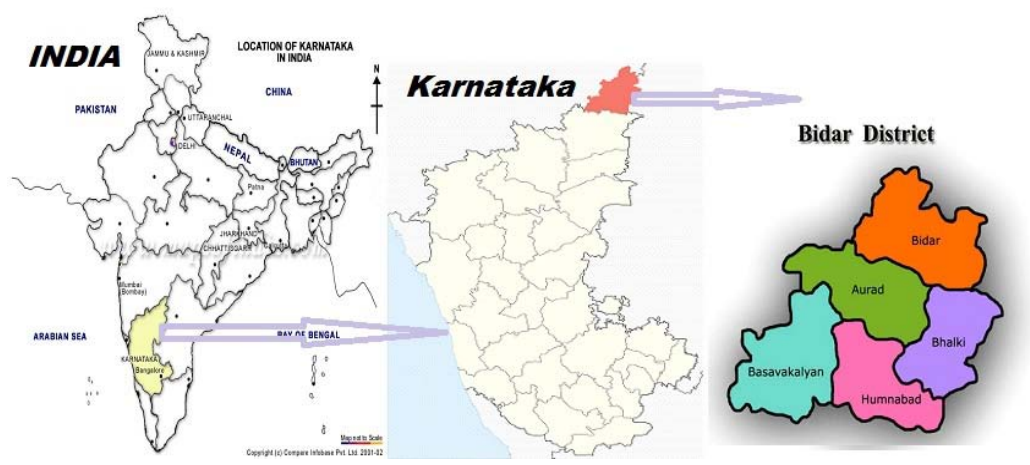

Fig. 1: Location map of Bidar district, Karnataka and India 


\section{Survey and documentation}

Surveys were undertaken at January 2018-July 2019 in villages, in ethnic people community and medicinal healers or local herbal practitioners in the study area. 42 medicinal healers' interviews were undertaken by the help of questioners or standard survey datasheet [7]. Through interviews and discussions, information about edible wild plants used for medicine to cure various diseases gathered. Plants were collect and herbarium specimens prepared. Plant specimens were identified with the help of floras and internet sources [8-11].

\section{RESULTS}

During the survey, a description of 63 species belonging to 40 families and 53 genera was recorded (fig. 2). The information was collect from different 42 medicinal healers by ages between $30-80$ y old (fig. 3). The description provides an account of these 63 species enumerate with regards the botanical name, family, Bidar local name/s, available season, part/s used preparation or dosage of medicine, precautions (if present) and ailments or diseases (table 1). Among 63 edible wild plants the plant parts used leaf ( 31 species) is most abundantly used followed by fruits (22 species) and other plant parts are with less than 5 species i.e. 4, 32 and single species (fig. 4). Plant materials were bringing from the forest in available seasons and make as tablet or other forms to treat the diseases. Sometimes fresh materials are used to treat for particular diseases.

Habit-wise distribution of medicinal, edible wild plants is shown in fig. 5. Herbs (22) are found to be the most used plants fallowed by trees (18), climbers (14) and shrubs (09) in descending order.

Cuccurbitaceae and Amaranthaceae were found to be the most common families with 7 and 6 species each, respectively. Other important families were Euphorbiaceae (4 species), Leguminaceae ( 4 species, in this family, 2 species of subfamily Caesalpinioideae and 2 species Mimosoideae), Aracaceae, Araceae, Chenopodiaceae, Ebenaceae, Moraceae, Portulacaceae, Rhamnaceae, Rutaceae and Solanaceae ( 2 species each) and rest of the 24 families had one wild edible medicinal species each (fig. 6).

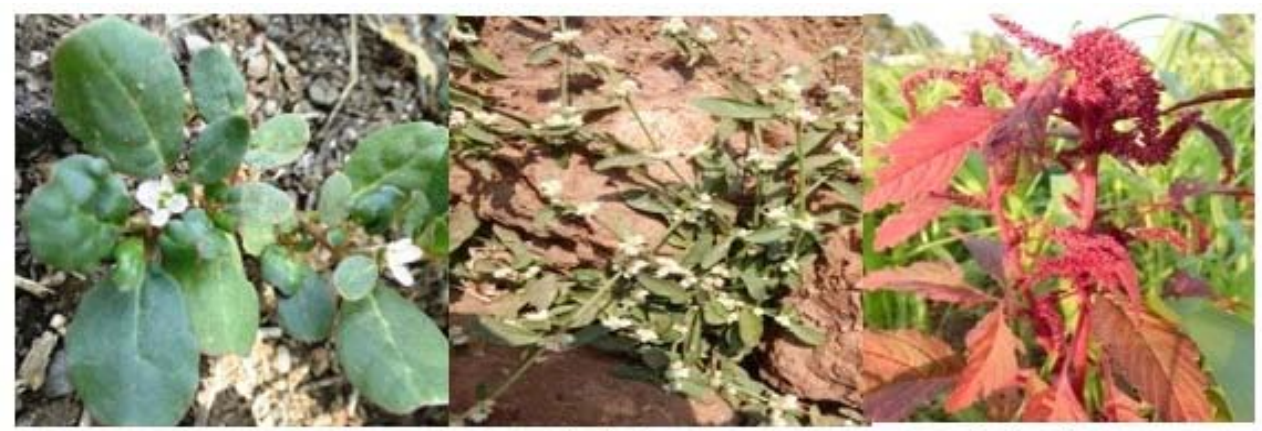

1. T. portulacastrum L. 2. A. sessilis (L.) R. Br. ex DC. 3. A. hybridus L.

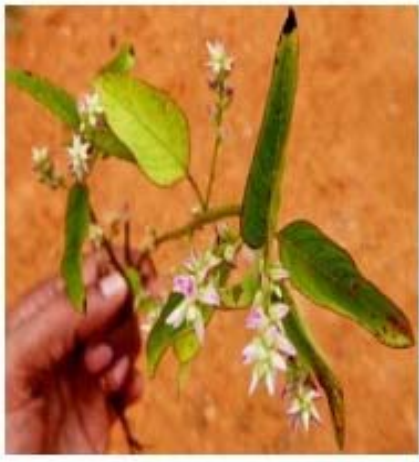

4. D. muricata (L.) Mart.,

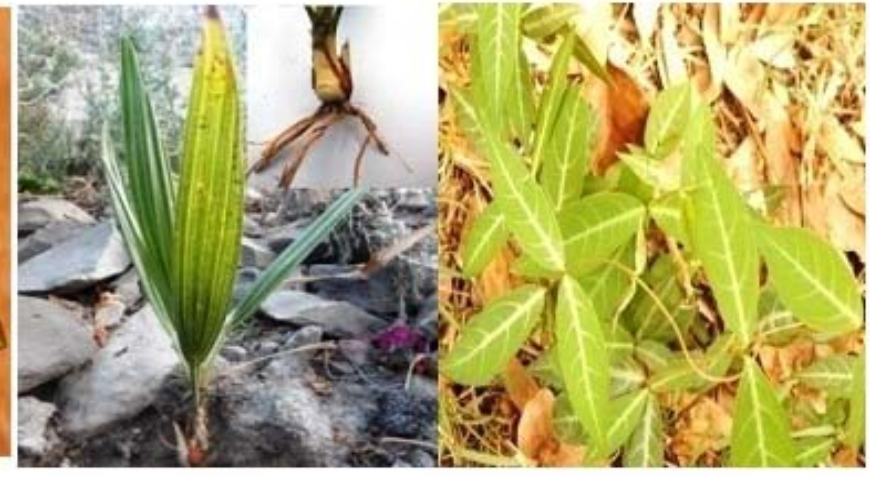

5. C.orchioides Gaertn. 6. H. indicus (L.) R. Br.,

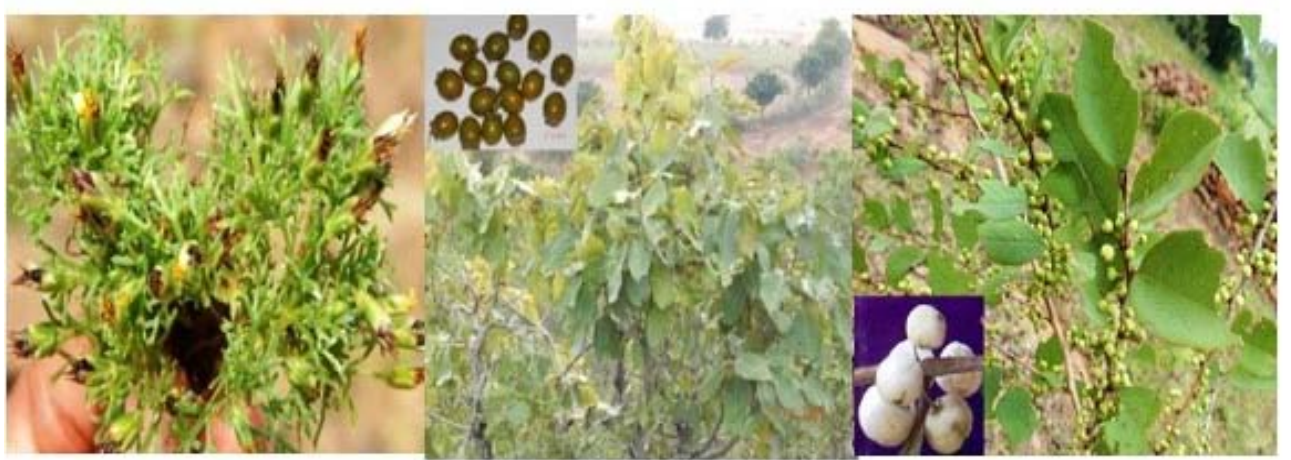

7. G. bosvallea DC. 8. D. melanoxylon Roxb. 9. F. leucopyrus Wild.

Fig. 2: Photographs of some reported plants of edible wild plants used as medicine 


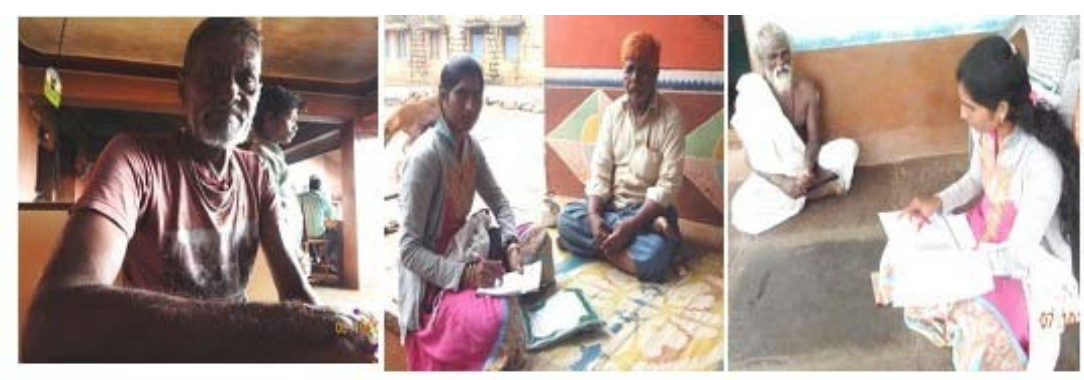

Anneppa Dhanagaar Vittal Maharaj Gundappa Muthyaa

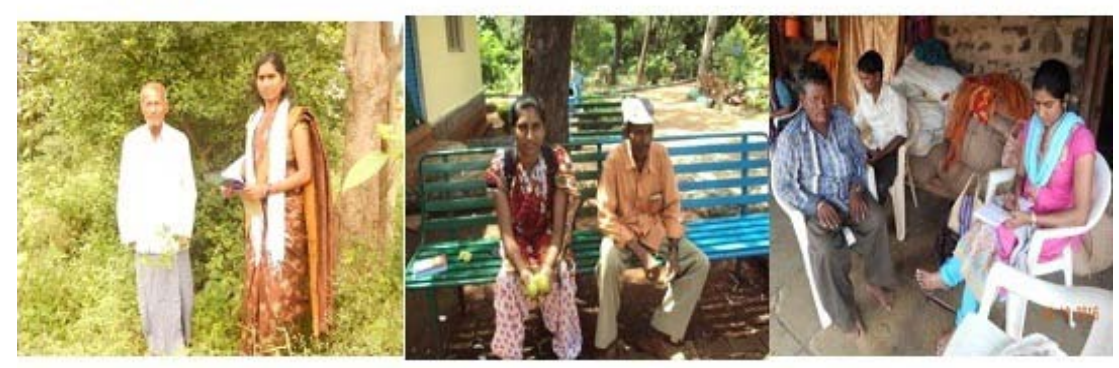

A.G. Shambu

Nisaar Ahammad

Mainoddhin Khan

Fig. 3: Few local traditional health practitioners in bidar district

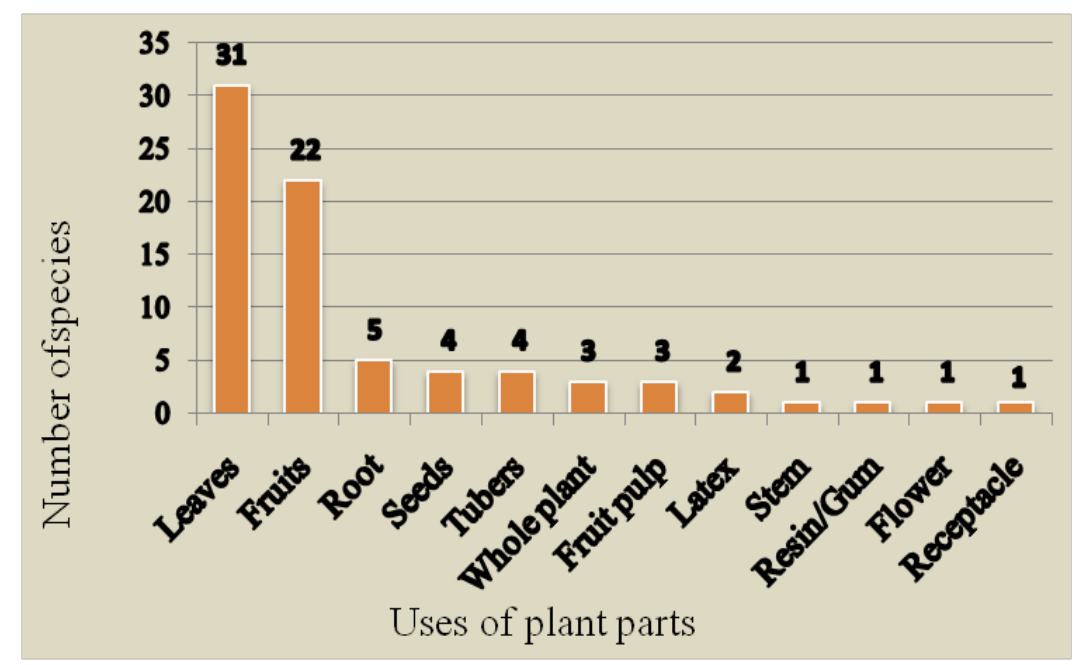

Fig. 4: Parts of the edible wild plants used as medicine in Bidar district

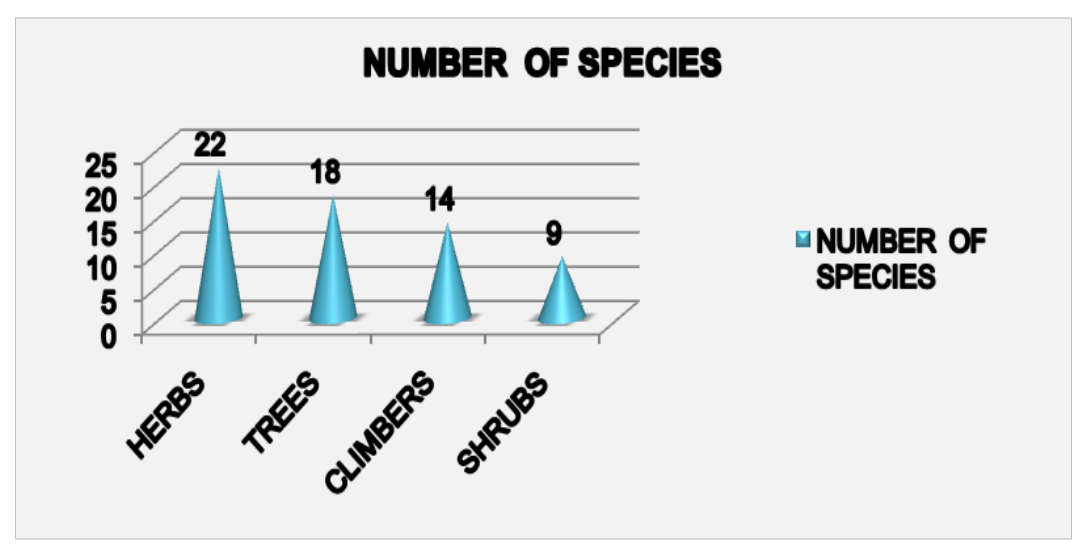

Fig. 5: Habit wise distribution of medicinal wild edible plants in Bidar district 


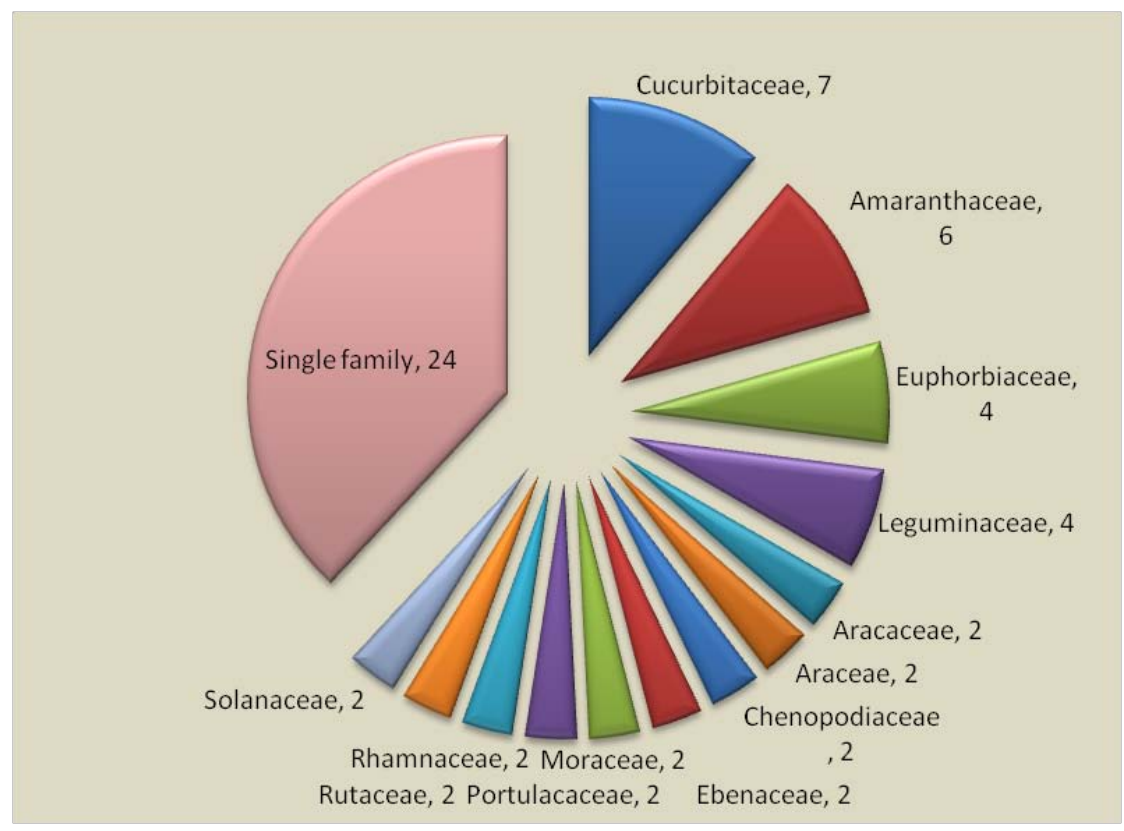

Fig. 6: Distribution of medicinal wild edible plants under various botanical families

\section{DISCUSSION}

The literature reveals that Trianthema portulacastrum root is used for killing the ascites. Plant is used as medicine for antiinflammatory and anti-carcinogenic activities $[12,13]$. The studies of ethnobotanical values of plants against 25 types of ailments were explored by using 31 medicinal plants belonging to 25 families in the people of Kailasakonda Sacred Grove [14]. Curculigo orchiodes and Amorphophallus paeoniifolius root and rhizome are used as medicine to treat various diseases such as abortifacient, asthma, cough, cuts, filaria, fever, itching, leucorrhoea, nose bleeding, piles, bleed, tonic, unconsciousness of children and wounds, earache, inflammation throat, respiratory complaints, pimples and piles [15]. Alternanthera sessilis, Amaranthus hybridus, A. spinosus, A. viridis, Digera muricata and Celosia argentea leaves cooked as vegetable and used as medicine for liver ailments, dysentery, diarrhea, dizziness, constipation, urinary disorder etc., and marketed in local markets [16-20]. Semecarpus anacardium leaf and fruit juice is used for colic, high blood pressure, stomachache sprains and rheumatism. Merremia gangetica root, leaves and the whole plant are used as purgative, diuretic, laxative, cough, headache, neuralgia, rheumatism, diuretic, inflammation, troubles of nose, fever and for the treatment of cancer, crude drug and other ayurvedic formulations [21-25]. Annona squomosa, Centella asiatica, Hemidesmus indicus, Cucumis trigonus and Momordica charantia seeds, root, leaves, fruits and the whole plant are used for to strengthen the bones, relieve vomiting, remove burning sensation, brain tonic, antibacterial, anti-diabetic, anti-fungal, etc [26-30]. The study reveals that some wild edible plants have dual role as food and medicinal in Palghar district. So, the total such 40 varieties of plant species are documented for phytochemical and nutraceutical analysis [31]

However, the large extent of ethnobotanical work on medicinal plants is carried out in worldwide, within the India and Karnataka state. In Bidar district medicinal healers are having lot of knowledge about Ethnobotany and ethnomedicine. There is no detailed study on documentation of edible wild plants used as medicine in the study area. So far, the present paper is gives detailed information of edible wild plants with their medicinal value for different ailments.

Table 1: List of medicinal properties of edible wild plants

\begin{tabular}{|c|c|c|c|c|c|c|c|c|}
\hline S. No. & $\begin{array}{l}\text { Botanical } \\
\text { name }\end{array}$ & $\begin{array}{l}\text { Family/Subfa } \\
\text { mily }\end{array}$ & $\begin{array}{l}\text { HGUG } \\
\text { voucher } \\
\text { no. }\end{array}$ & $\begin{array}{l}\text { Bidar local } \\
\text { name } / \mathrm{s}\end{array}$ & $\begin{array}{l}\text { Available } \\
\text { season }\end{array}$ & Part/s used & Preparation/dosage & Ailment/s or disease/s \\
\hline 1. & $\begin{array}{l}\text { Trianthema } \\
\text { portulacastru } \\
m \mathrm{~L} .\end{array}$ & Aizoceae & 5216 & Khappur palya & Rainy & Leaves & $\begin{array}{l}\text { Leaves grinds with salt } \\
\text { then apply on the } \\
\text { wound caused by spine }\end{array}$ & Anti inflammatory \\
\hline 2. & $\begin{array}{l}\text { Alternanthera } \\
\text { sessilis (L.) R. } \\
\text { Br. ex DC. }\end{array}$ & Amaranthaceae & 5139 & Garundi palya & Rainy & Leaves & $\begin{array}{l}\text { Leaves used as } \\
\text { vegetable }\end{array}$ & Piles, Osteomalacia \\
\hline 3. & $\begin{array}{l}\text { Amaranthus } \\
\text { hybridus L. }\end{array}$ & Amaranthaceae & 5140 & $\begin{array}{l}\text { Rajgiri palya, } \\
\text { Shravani math }\end{array}$ & Rainy & Leaves & $\begin{array}{l}\text { Leaves used as } \\
\text { vegetable }\end{array}$ & $\begin{array}{l}\text { Digestive, stomach disorders, } \\
\text { purgative, dysmenorrhoea, } \\
\text { piles }\end{array}$ \\
\hline 4. & $\begin{array}{l}\text { Amaranthus } \\
\text { spinosus } \mathrm{L} .\end{array}$ & Amaranthaceae & 5141 & Kirgasale palya & Rainy & Leaves & $\begin{array}{l}\text { Leaves used as } \\
\text { vegetable }\end{array}$ & $\begin{array}{l}\text { Anaemia, Osteomalacia, fever, } \\
\text { colour blindness, jaundice, } \\
\text { kidney stones }\end{array}$ \\
\hline 5. & $\begin{array}{l}\text { Amaranthus } \\
\text { viridis } \mathrm{L} .\end{array}$ & Amaranthaceae & 5143 & $\begin{array}{l}\text { Sanna rajgiri } \\
\text { playa, kantha } \\
\text { bhaji }\end{array}$ & Rainy & $\begin{array}{l}\text { Young } \\
\text { shoots, } \\
\text { Leaves }\end{array}$ & $\begin{array}{l}\text { Young shoots or leaves } \\
\text { used as vegetable }\end{array}$ & $\begin{array}{l}\text { Digestive, Purgative, stomach } \\
\text { disorders, Anaemia }\end{array}$ \\
\hline 6. & $\begin{array}{l}\text { Celosia } \\
\text { argentea } \mathrm{L} .\end{array}$ & Amaranthaceae & 5159 & $\begin{array}{l}\text { Kukken gida, } \\
\text { Anne soppu, } \\
\text { Karada }\end{array}$ & Winter & Leaves & $\begin{array}{l}\text { Leaves eaten with rock } \\
\text { sugar. } \\
\text { Leaves used as } \\
\text { vegetable }\end{array}$ & $\begin{array}{l}\text { Cooling agent, improve } \\
\text { stamina, } \\
\text { Mouth ulcer, colour blindness }\end{array}$ \\
\hline 7. & $\begin{array}{l}\text { Digera } \\
\text { muricata (L.) } \\
\text { Mart. }\end{array}$ & Amaranthaceae & 5169 & $\begin{array}{l}\text { Gundagarji } \\
\text { palya, } \\
\text { Gudeli palya }\end{array}$ & Rainy & Young plant & $\begin{array}{l}\text { Young plant used as } \\
\text { vegetable }\end{array}$ & Hepatitis, digestion, diuretic \\
\hline
\end{tabular}




\begin{tabular}{|c|c|c|c|c|c|c|c|c|}
\hline S. No. & $\begin{array}{l}\text { Botanical } \\
\text { name }\end{array}$ & $\begin{array}{l}\text { Family/Subfa } \\
\text { mily }\end{array}$ & $\begin{array}{l}\text { HGUG } \\
\text { voucher } \\
\text { no. }\end{array}$ & $\begin{array}{l}\text { Bidar local } \\
\text { name } / \mathrm{s}\end{array}$ & $\begin{array}{l}\text { Available } \\
\text { season }\end{array}$ & Part/s used & Preparation/dosage & Ailment/s or disease/s \\
\hline 8. & $\begin{array}{l}\text { Curculigo } \\
\text { orchioides } \\
\text { Gaertn. }\end{array}$ & Amaryllidaceae & 5168 & $\begin{array}{l}\text { Khaduri, } \\
\text { Adi ulagadde, } \\
\text { One padarin } \\
\text { ulagadde, Kali } \\
\text { musli }\end{array}$ & Rainy & Tuber, fruits & $\begin{array}{l}\text { Tuber of Curculigo } \\
\text { orchioides and Castor } \\
\text { leaf powder makes a } \\
\text { tablet, take daily for } 21 \\
\text { d with empty stomach } \\
\text { Ripe fruits eaten raw } \\
\text { to enhance immunity }\end{array}$ & $\begin{array}{l}\text { Jaundice, strengthen } \\
\text { immunity } \\
\text { Strengthen immunity, blood } \\
\text { purification }\end{array}$ \\
\hline 9. & $\begin{array}{l}\text { Semecarpus } \\
\text { anacardium } \mathrm{L} .\end{array}$ & Anacardiaceae & 5210 & $\begin{array}{l}\text { Kyare kai, } \\
\text { Bibha, Geru }\end{array}$ & Winter & $\begin{array}{l}\text { Receptacle, } \\
\text { Fruit, } \\
\text { Flower }\end{array}$ & $\begin{array}{l}\text { Boil the receptacles } \\
\text { with pigeon pea and } \\
\text { eat than drink only a } \\
\text { cup of water to cure. } \\
\text { Burn the receptacle to } \\
\text { produce oil than rubs } \\
\text { the oil on throat. } \\
\text { Eat flower daily } \\
\text { morning up to a week }\end{array}$ & $\begin{array}{l}\text { Heart attack, Heart diseases } \\
\text { Wet cough or whooping } \\
\text { cough } \\
\text { Whooping cough }\end{array}$ \\
\hline 10. & $\begin{array}{l}\text { Annona } \\
\text { squamosa L. }\end{array}$ & Annonaceae & 5146 & Sithaphal & Winter & Fruits, Seeds & $\begin{array}{l}\text { Fruit epicarp paste } \\
\text { applied on wound } \\
\text { Seeds powder eaten } \\
\text { with empty stomach }\end{array}$ & $\begin{array}{l}\text { Digestive, fungal infections, } \\
\text { ringworm, psoriasis, } \\
\text { leptospirosis } \\
\text { Killing the earthworms in } \\
\text { stomach, stomach ache }\end{array}$ \\
\hline 11. & $\begin{array}{l}\text { Centella } \\
\text { asiatica (L.) } \\
\text { Urb. }\end{array}$ & Apiaceae & 5160 & $\begin{array}{l}\text { Brahmi, } \\
\text { Ondelage }\end{array}$ & Rainy & Leaves & $\begin{array}{l}\text { Leaves chewed and } \\
\text { eaten }\end{array}$ & $\begin{array}{l}\text { Memory enhancer, kidney } \\
\text { stones }\end{array}$ \\
\hline 12. & $\begin{array}{l}\text { Hemidesmus } \\
\text { indicus (L.) R. } \\
\text { Br. }\end{array}$ & Apocynaceae & 5184 & $\begin{array}{l}\text { Sugandi beru, } \\
\text { Halibarin gida }\end{array}$ & Rainy & Root & $\begin{array}{l}\text { Root powder applied } \\
\text { Root powder boil and } \\
\text { drink }\end{array}$ & $\begin{array}{l}\text { Skin infection, feet cracks, } \\
\text { toothache } \\
\text { Blood purifier }\end{array}$ \\
\hline 13. & $\begin{array}{l}\text { Phoenix } \\
\text { sylvestris (L.) } \\
\text { Roxb. }\end{array}$ & Aracaceae & 5200 & $\begin{array}{l}\text { Shindi kai, } \\
\text { Enchalu hannu }\end{array}$ & $\begin{array}{l}\text { Throughou } \\
t \text { the year }\end{array}$ & Seeds, Root & $\begin{array}{l}\text { Seeds eaten raw } \\
\text { Cattle can feed bulging } \\
\text { roots with above } \\
\text { ground at the base }\end{array}$ & $\begin{array}{l}\text { Strengthen immunity, } \\
\text { Milking in Cattle }\end{array}$ \\
\hline 14. & $\begin{array}{l}\text { Borassus } \\
\text { flabellifer } \mathrm{L} .\end{array}$ & Aracaceae & 5151 & Taadin Kai & Winter & Fruits & Ripe fruits eaten raw & Laxative, stimulant \\
\hline 15. & $\begin{array}{l}\text { Amorphophall } \\
\text { us } \\
\text { paeoniifolius } \\
\text { (Dennst.) } \\
\text { Nicolson }\end{array}$ & Araceae & 5144 & $\begin{array}{l}\text { Suvarna gadde, } \\
\text { Havald gadde, } \\
\text { Sonevell }\end{array}$ & Summer & Tubers & Eat raw tubers 3-4 d & Piles, menstrual cycle regular \\
\hline 16. & $\begin{array}{l}\text { Colacasia } \\
\text { esculenta (L.) } \\
\text { Schott. }\end{array}$ & Araceae & 5165 & Shavi palya & Rainy & $\begin{array}{l}\text { Leaves, } \\
\text { Tubers }\end{array}$ & $\begin{array}{l}\text { Take } 2 \text { rupees coin size } \\
\text { leaf of Colacasia } \\
\text { esculenta smashed and } \\
\text { drink within cow milk } \\
\text { or mother milk } \\
\text { Tubers and leaves are } \\
\text { used as vegetable, } \\
\text { snacks and sauce. }\end{array}$ & $\begin{array}{l}\text { Dry cough, whooping cough, } \\
\text { Pneumonia, Blood } \\
\text { purification, Digestion. } \\
\text { Kidney stone, Urinary } \\
\text { disorder, stomach ache, piles. }\end{array}$ \\
\hline 17. & $\begin{array}{l}\text { Glossocardia } \\
\text { bosvallea (L. } \\
\text { f.) DC. }\end{array}$ & Asteraceae & 5185 & Adi sabski & Rainy & Whole plant & $\begin{array}{l}\text { Whole plant used as } \\
\text { vegetable }\end{array}$ & Strengthen immunity, fever \\
\hline 18. & $\begin{array}{l}\text { Opuntia } \\
\text { stricta (Haw.) } \\
\text { Haworth. }\end{array}$ & Cactaceae & 5197 & $\begin{array}{l}\text { Nagarpaden } \\
\text { gida }\end{array}$ & Winter & Leaves & $\begin{array}{l}\text { Leaf (Phyllode) cut into } \\
\text { two equal portion } \\
\text { warm and apply on } \\
\text { chest tumour }\end{array}$ & Lump's, cancer \\
\hline 19. & $\begin{array}{l}\text { Capparis } \\
\text { zeylanica } \mathrm{L} .\end{array}$ & Capparaceae & 5155 & Tottilu belli & Winter & Leaves & $\begin{array}{l}\text { Eat } 3-4 \mathrm{~d} 50 \mathrm{~g} \text { of leaf } \\
\text { extract mix with ghee } \\
\text { and sugar or Jaggary } \\
\text { Leaves mixed with salt } \\
\text { and brushed teeth to } \\
\text { cure the toothache. }\end{array}$ & $\begin{array}{l}\text { Menstrual problems, } \\
\text { gynaecological disorder, } \\
\text { stomachic } \\
\text { Toothache, stomach ache }\end{array}$ \\
\hline 20. & Basella alba L. & $\begin{array}{l}\text { Chenopodiacea } \\
\text { e }\end{array}$ & 5150 & $\begin{array}{l}\text { Basale soppu, } \\
\text { Bansali }\end{array}$ & Rainy & Leaves & $\begin{array}{l}\text { 1-2 Leaves eaten with } \\
\text { empty stomach in early } \\
\text { morning }\end{array}$ & $\begin{array}{l}\text { Constipation, Anaemia, } \\
\text { digestive, appetite }\end{array}$ \\
\hline 21. & $\begin{array}{l}\text { Chenopodium } \\
\text { album } \mathrm{L} .\end{array}$ & $\begin{array}{l}\text { Chenopodiacea } \\
\text { e }\end{array}$ & 5161 & $\begin{array}{l}\text { Chukka, } \\
\text { Hunachikki }\end{array}$ & Winter & Leaves & $\begin{array}{l}\text { Leaves used as a } \\
\text { vegetable }\end{array}$ & $\begin{array}{l}\text { Cooling agent, stomach pain, } \\
\text { kidney stone, yellow jaundice, } \\
\text { piles }\end{array}$ \\
\hline 22. & $\begin{array}{l}\text { Merremia } \\
\text { emarginata } \\
\text { (Burm. f.) } \\
\text { Hallier f. }\end{array}$ & Convolvulaceae & 5191 & Ilikiwi gida & Rainy & $\begin{array}{l}\text { Leaves, } \\
\text { Whole plant }\end{array}$ & $\begin{array}{l}\text { Leaves used as } \\
\text { vegetable } \\
\text { One spoon of whole } \\
\text { plant decoction given } \\
\text { to children daily at one } \\
\text { time }\end{array}$ & $\begin{array}{l}\text { Kidney stones, heart diseases, } \\
\text { urinary diseases, digestive, } \\
\text { diuretic. } \\
\text { Urine problems, liver } \\
\text { problems }\end{array}$ \\
\hline 23. & $\begin{array}{l}\text { Kalanchoe } \\
\text { pinnata } \\
\text { (Lam.) Pers. }\end{array}$ & Crassulaceae & 5185 & $\begin{array}{l}\text { Tilval, } \\
\text { Gandukalinga }\end{array}$ & Summer & Leaves & $\begin{array}{l}\text { Leaves used eaten raw } \\
\text { to treat }\end{array}$ & Asthma, cough \\
\hline 24. & $\begin{array}{l}\text { Coccinia } \\
\text { indica Wight } \\
\text { and Arn. }\end{array}$ & Cucurbitaceae & 5163 & Tonde kai & Winter & Fruits & $\begin{array}{l}\text { Chew } 1 \text { or } 2 \text { unripe } \\
\text { fruits }\end{array}$ & Mouth ulcer, fever \\
\hline 25. & $\begin{array}{l}\text { Cucumis } \\
\text { trigonus Roxb. }\end{array}$ & Cucurbitaceae & 5167 & $\begin{array}{l}\text { Gudumin kai, } \\
\text { Kaadu sauté kai }\end{array}$ & Winter & Fruits & Fruits eaten raw & $\begin{array}{l}\text { Cooling agent, Acidity, } \\
\text { diabetes, digestive }\end{array}$ \\
\hline 26. & $\begin{array}{l}\text { Diplocyclos } \\
\text { palmatus (L.) }\end{array}$ & Cucurbitaceae & 5175 & $\begin{array}{l}\text { Shivalingi, } \\
\text { sumblen kai }\end{array}$ & Rainy & Seeds & $\begin{array}{l}\text { Seeds powder mixed } \\
\text { with cow milk and }\end{array}$ & $\begin{array}{l}\text { Fertility in women, prevent } \\
\text { miscarriage }\end{array}$ \\
\hline
\end{tabular}




\begin{tabular}{|c|c|c|c|c|c|c|c|c|}
\hline S. No. & $\begin{array}{l}\text { Botanical } \\
\text { name }\end{array}$ & $\begin{array}{l}\text { Family/Subfa } \\
\text { mily }\end{array}$ & $\begin{array}{l}\text { HGUG } \\
\text { voucher } \\
\text { no. }\end{array}$ & $\begin{array}{l}\text { Bidar local } \\
\text { name } / \mathrm{s}\end{array}$ & $\begin{array}{l}\text { Available } \\
\text { season }\end{array}$ & Part/s used & Preparation/dosage & Ailment/s or disease/s \\
\hline & C. Jeffrey. & & & & & & drink & \\
\hline 27. & $\begin{array}{l}\text { Momordica } \\
\text { charantia L. }\end{array}$ & Cucurbitaceae & 5192 & Haagal kai & Rainy & Fruits & $\begin{array}{l}\text { Drink a cup of fruit } \\
\text { juice for } 2-3 \mathrm{~d} \\
\text { Fruits eaten raw }\end{array}$ & $\begin{array}{l}\text { Diabetes, pitta } \\
\text { Acidity }\end{array}$ \\
\hline 28. & $\begin{array}{l}\text { Momordica } \\
\text { dioica Roxb. } \\
\text { Ex Willd. }\end{array}$ & Cucurbitaceae & 5194 & Midi hagul kai & Rainy & $\begin{array}{l}\text { Leaves, } \\
\text { Fruits }\end{array}$ & $\begin{array}{l}\text { Drink leaf or fruit juice } \\
2-3 \mathrm{~d} \\
\text { Fruits used as vegetable } \\
\text { Fruits used as vegetable }\end{array}$ & $\begin{array}{l}\text { Diabetes, fever } \\
\text { Acidity, Pitta }\end{array}$ \\
\hline 29. & $\begin{array}{l}\text { Momordica } \\
\text { cymbalaria } \\
\text { Hook. f. }\end{array}$ & Cucurbitaceae & 5193 & Karchi kai & Rainy & $\begin{array}{l}\text { Fruits, } \\
\text { Tubers, } \\
\text { Leaves }\end{array}$ & $\begin{array}{l}\text { Fruits used as vegetable } \\
\text { Tubers eaten raw to } \\
\text { treat } \\
1-2 \text { leaves eaten at an } \\
\text { early morning }\end{array}$ & $\begin{array}{l}\text { Strengthen immunity } \\
\text { Diabetes } \\
\text { Pitta }\end{array}$ \\
\hline 30. & $\begin{array}{l}\text { Mukia } \\
\text { maderaspatan } \\
\text { a (L.) M. } \\
\text { Roem. }\end{array}$ & Cucurbitaceae & 5196 & $\begin{array}{l}\text { Yemmighogari } \\
\text { belli, Benneti }\end{array}$ & Rainy & Root, Fruits & $\begin{array}{l}\text { Root extract applied on } \\
\text { skin and pimples. } \\
\text { Fruits eaten raw }\end{array}$ & Skin diseases \\
\hline 31. & $\begin{array}{l}\text { Diospyrous } \\
\text { melanoxylon } \\
\text { Roxb. }\end{array}$ & Ebenaceae & 5173 & $\begin{array}{l}\text { Tendu, } \\
\text { Thumbri, } \\
\text { Illinta }\end{array}$ & Rainy & Fruits & $\begin{array}{l}\text { Fruits eaten daily early } \\
\text { morning with empty } \\
\text { stomach }\end{array}$ & $\begin{array}{l}\text { Cooling agent, Acidity, } \\
\text { stomach problems }\end{array}$ \\
\hline 32. & $\begin{array}{l}\text { Diospyrous } \\
\text { montana } \\
\text { Roxb. }\end{array}$ & Ebenaceae & 5174 & $\begin{array}{l}\text { Enchil hannu, } \\
\text { Timbru }\end{array}$ & Summer & Fruits & $\begin{array}{l}\text { Fruit eaten daily early } \\
\text { morning with empty } \\
\text { stomach }\end{array}$ & Cooling agent, Acidity \\
\hline 33. & $\begin{array}{l}\text { Phyllanthus } \\
\text { acidus (L.) } \\
\text { Skeels. }\end{array}$ & Euphorbiaceae & 5201 & $\begin{array}{l}\text { Havla, Keeri } \\
\text { nelli, Gundi } \\
\text { nelli }\end{array}$ & Summer & Fruits & $\begin{array}{l}\text { Equal amount of }(1 / 2 \\
\text { tsp.) Phyllanthus acidus } \\
\text { fruit powder+Celosia } \\
\text { argentia leaf } \\
\text { powder+Croton tiglium } \\
\text { seed powder mixed } \\
\text { well and take orally up } \\
\text { to } 15-20 \mathrm{~d} \text {. } \\
\text { Fruits eaten raw with } \\
\text { salt }\end{array}$ & $\begin{array}{l}\text { Blood purification, appetite. } \\
\text { Digestive, pitta, giddiness, } \\
\text { omitting, acidity }\end{array}$ \\
\hline 34. & $\begin{array}{l}\text { Emblica } \\
\text { officinalis } \\
\text { Gaertn. }\end{array}$ & Euphorbiaceae & 5202 & $\begin{array}{l}\text { Nelle kai, Amla, } \\
\text { Dodda nelli kai }\end{array}$ & Winter & $\begin{array}{l}\text { Fruits, } \\
\text { Leaves }\end{array}$ & $\begin{array}{l}\text { Take fruit powder of } \\
\text { Phyllanthus emblica, } \\
\text { Terminalia bellarica } \\
\text { and Terminalia chebula } \\
\text { in honey ( } 50 \%) \text { and } \\
\text { Allium sativum paste } \\
(25 \%) \text {. } \\
\text { Leaves extract applied } \\
\text { on wounds }\end{array}$ & $\begin{array}{l}\text { Control high blood pressure, } \\
\text { cough and acidity, stomach } \\
\text { problems } \\
\text { Mouth ulcer }\end{array}$ \\
\hline 35. & $\begin{array}{l}\text { Kirganalia } \\
\text { reticulata } \\
\text { (Poir.) Baill. }\end{array}$ & Euphorbiaceae & 5186 & $\begin{array}{l}\text { Kempu huli, } \\
\text { Huli hannu }\end{array}$ & Summer & Leaves & $\begin{array}{l}\text { Leaves extract of } \\
\text { Kirganalia reticulata } \\
\text { and leaves extract of } \\
\text { Flueggea leucopyrus } \\
\text { mixed in buttermilk } \\
\text { and take orally for } 7 \mathrm{~d}\end{array}$ & $\begin{array}{l}\text { Red discharge, } \\
\text { White discharge }\end{array}$ \\
\hline 36. & $\begin{array}{l}\text { Flueggea } \\
\text { leucopyrus } \\
\text { Wild. }\end{array}$ & Euphorbiaceae & 5209 & Bili huli gida & Winter & Leaves & $\begin{array}{l}\text { Leaves extract mixed } \\
\text { in drinking water or } \\
\text { cow milk or goat milk } \\
\text { and take orally for } 7 \mathrm{~d}\end{array}$ & White discharge \\
\hline 37. & $\begin{array}{l}\text { Plectranthus } \\
\text { amboinicus } \\
\text { (Lour.) } \\
\text { Spreng. }\end{array}$ & Lamiaceae & 5205 & $\begin{array}{l}\text { Ajeevan tappul, } \\
\text { Dhapati yeli }\end{array}$ & $\begin{array}{l}\text { Throughou } \\
\text { t the year }\end{array}$ & Leaves & $\begin{array}{l}\text { Leaves used in meals } \\
\text { Take leaves extract of } \\
\text { Occimum sanctum and } \\
\text { Plectranthus } \\
\text { amboinicus in honey. }\end{array}$ & $\begin{array}{l}\text { Digestive, hepatitis, diuretic, } \\
\text { laxative, cough, asthma. } \\
\text { Cold, cough in children }\end{array}$ \\
\hline 38. & $\begin{array}{l}\text { Cassia fistula } \\
\text { L. }\end{array}$ & $\begin{array}{l}\text { Leguminaceae/C } \\
\text { aesalpinioideae }\end{array}$ & 5157 & Kakke hanuu & Summer & Root & $\begin{array}{l}\text { Root juice is applied on } \\
\text { the snake bite }\end{array}$ & Snack bite \\
\hline 39. & $\begin{array}{l}\text { Senna tora } \\
\text { (L.) Roxb. }\end{array}$ & $\begin{array}{l}\text { Leguminaceae/C } \\
\text { aesalpinioideae }\end{array}$ & 5158 & Chagachi gida & Winter & $\begin{array}{l}\text { Seeds } \\
\text { Leaves }\end{array}$ & $\begin{array}{l}\text { Seeds eaten raw and } \\
\text { used as vegetable } \\
\text { Leaves used as } \\
\text { vegetable }\end{array}$ & $\begin{array}{l}\text { Skin diseases, edema } \\
\text { (Swelling of the body), } \\
\text { enhance milk in cattle } \\
\text { Strengthen the immunity, } \\
\text { enhance milk in cattle } \\
\text { Plant is consists of omega } 3 \\
\text { fatty acids }\end{array}$ \\
\hline 40. & $\begin{array}{l}\text { Acacia } \\
\text { arabica } \\
\text { (Lam.) Willd. }\end{array}$ & $\begin{array}{l}\text { Leguminaceae/ } \\
\text { Mimosoideae }\end{array}$ & 5137 & $\begin{array}{l}\text { Babli gida, Kari } \\
\text { jaali }\end{array}$ & $\begin{array}{l}\text { Throughou } \\
t \text { the year }\end{array}$ & Resin/Gum & $\begin{array}{l}\text { Resin or gum eaten } \\
\text { raw or cooked. } \\
\text { Women eaten after } \\
\text { maturation (Menstrual } \\
\text { Cycle time) and child } \\
\text { birth (Delivery) }\end{array}$ & Strengthen immunity \\
\hline 41. & $\begin{array}{l}\text { Pithecellobiu } \\
\text { m dulce } \\
\text { (Roxb.) Benth. }\end{array}$ & $\begin{array}{l}\text { Leguminaceae/ } \\
\text { Mimosoideae }\end{array}$ & 5204 & $\begin{array}{l}\text { Gorak kaambli, } \\
\text { Kodabyale kai, } \\
\text { Hulihunase }\end{array}$ & Summer & Fruits & $\begin{array}{l}\text { Ripped fruits eaten } \\
\text { raw }\end{array}$ & Cooling agent, acidity contro \\
\hline 42. & $\begin{array}{l}\text { Asparagus } \\
\text { racemosus } \\
\text { Willd. }\end{array}$ & Lilliaceae & 5147 & $\begin{array}{l}\text { Halavu makkal } \\
\text { taayi, Shatavari }\end{array}$ & $\begin{array}{l}\text { Throughou } \\
\text { t the year }\end{array}$ & Tubers & $\begin{array}{l}\text { Powder of Asparagus } \\
\text { racemosus } \\
\text { tuber+Diplocyclos } \\
\text { palmatus fruit+cumin } \\
\text { powder mixed in honey } \\
\text { and eat } 3 \mathrm{~d} \text { during M C }\end{array}$ & $\begin{array}{l}\text { Strengthen immunity, } \\
\text { antipyretic, menstrual } \\
\text { problems } \\
\text { Sterility, dysentery }\end{array}$ \\
\hline
\end{tabular}




\begin{tabular}{|c|c|c|c|c|c|c|c|c|}
\hline S. No. & $\begin{array}{l}\text { Botanical } \\
\text { name }\end{array}$ & $\begin{array}{l}\text { Family/Subfa } \\
\text { mily }\end{array}$ & $\begin{array}{l}\text { HGUG } \\
\text { voucher } \\
\text { no. }\end{array}$ & $\begin{array}{l}\text { Bidar local } \\
\text { name } / \mathrm{s}\end{array}$ & $\begin{array}{l}\text { Available } \\
\text { season }\end{array}$ & Part/s used & Preparation/dosage & Ailment/s or disease $/ \mathrm{s}$ \\
\hline & & & & & & & $\begin{array}{l}\text { period } \\
\text { (Precaution: Eat only } \\
\text { white } \\
\text { rice and curd for } 3 \mathrm{~d} \text { ) } \\
\text { Tubers used as } \\
\text { vegetable }\end{array}$ & \\
\hline 43. & $\begin{array}{l}\text { Abelmoschus } \\
\text { moschatus } \\
\text { Medik. }\end{array}$ & Malvaceae & 5136 & Advi bendekai & Winter & Fruits & $\begin{array}{l}\text { Fruits used as } \\
\text { vegetable during } \\
\text { suffering from diseases }\end{array}$ & $\begin{array}{l}\text { Strengthen immunity, } \\
\text { digestive, stomach ache, } \\
\text { constipation }\end{array}$ \\
\hline 44. & $\begin{array}{l}\text { Cocculus } \\
\text { hirsutus (L.) } \\
\text { Diels. }\end{array}$ & $\begin{array}{l}\text { Menispermacea } \\
\text { e }\end{array}$ & 5164 & $\begin{array}{l}\text { Dhangadgen } \\
\text { gida }\end{array}$ & Rainy & Leaves & $\begin{array}{l}\text { 2-3 Leaves used to } \\
\text { eaten daily morning }\end{array}$ & Male sterility \\
\hline 45. & $\begin{array}{l}\text { Ficus } \\
\text { racemosa L. }\end{array}$ & Moraceae & 5178 & Atti kai & Winter & Latex, Root & $\begin{array}{l}\text { Latex mixed with sugar } \\
\text { or batasha. } \\
\text { Root powder mixed in } \\
\text { pot water overnight } \\
\text { and drunk early in the } \\
\text { morning } \\
\text { Latex applied on } \\
\text { wounds }\end{array}$ & $\begin{array}{l}\text { Menstrual problems } \\
\text { Chest tumour, wounds. }\end{array}$ \\
\hline 46. & $\begin{array}{l}\text { Ficus hispida } \\
\text { L. }\end{array}$ & Moraceae & 5177 & Nela atti kai & Rainy & Latex & $\begin{array}{l}\text { Latex applied on skin } \\
\text { diseases and wounds }\end{array}$ & $\begin{array}{l}\text { Skin infections-pimples, } \\
\text { ringworm, wounds }\end{array}$ \\
\hline 47. & $\begin{array}{l}\text { Syzygium } \\
\text { cumini (L.) } \\
\text { Skeels. }\end{array}$ & Myrtaceae & 5214 & $\begin{array}{l}\text { Nerale hannu, } \\
\text { Kaala jamun, } \\
\text { Jaamun }\end{array}$ & Rainy & Fruits, Seeds & $\begin{array}{l}\text { Ripped fruits eaten } \\
\text { raw } \\
\text { Eaten fruits with seeds } \\
\text { Take a spoon of shade } \\
\text { dried seeds powder } \\
\text { daily early in the } \\
\text { morning }\end{array}$ & $\begin{array}{l}\text { High diabetes } \\
\text { Killing the earthworms in } \\
\text { stomach } \\
\text { Diabetes }\end{array}$ \\
\hline 48. & $\begin{array}{l}\text { Ximenia } \\
\text { americana } \mathrm{L} .\end{array}$ & Olacaceae & 5218 & $\begin{array}{l}\text { Nagari hannu, } \\
\text { Nakkeru }\end{array}$ & Summer & Leaves & $\begin{array}{l}\text { Take a glass of leaf } \\
\text { extract }\end{array}$ & Snack bite \\
\hline 49. & $\begin{array}{l}\text { Portulaca } \\
\text { oleracea } \mathrm{L} .\end{array}$ & Portulacaceae & 5206 & Dodda ghooli & Rainy & Leaves & $\begin{array}{l}\text { Leaves used as } \\
\text { vegetable }\end{array}$ & $\begin{array}{l}\text { Treatment for liver, kidney, } \\
\text { jaundice, constipation, } \\
\text { asthma }\end{array}$ \\
\hline 50. & $\begin{array}{l}\text { Portuluca } \\
\text { quadrifida } \mathrm{L} .\end{array}$ & Portulacaceae & 5207 & Sanna ghooli & Rainy & Whole plant & $\begin{array}{l}\text { Whole plant used as } \\
\text { vegetable }\end{array}$ & $\begin{array}{l}\text { Cooling agent, digestive, } \\
\text { diuretic }\end{array}$ \\
\hline 51. & $\begin{array}{l}\text { Ziziphus } \\
\text { mauritiana } \\
\text { Lam. }\end{array}$ & Rhamnaceae & 5219 & Bare hannu & Winter & $\begin{array}{l}\text { Spines, } \\
\text { Leaves }\end{array}$ & $\begin{array}{l}\text { Take } 3 \text { spines of the } \\
\text { plant and mark round } \\
\text { on wound or ring } \\
\text { gourd. } \\
\text { Leaf paste applied on } \\
\text { burns }\end{array}$ & $\begin{array}{l}\text { Fungal infections, } \\
\text { Burns }\end{array}$ \\
\hline 52. & $\begin{array}{l}\text { Ziziphus } \\
\text { oenoplia (L.) } \\
\text { Mill. }\end{array}$ & Rhamnaceae & 5221 & $\begin{array}{l}\text { Kaalaboor, Kari } \\
\text { bare kai }\end{array}$ & Winter & Fruits & $\begin{array}{l}\text { Ripped fruits eaten } \\
\text { raw }\end{array}$ & Jaundice, stomach problems \\
\hline 53. & $\begin{array}{l}\text { Gardenia } \\
\text { resinifera } \\
\text { Roth. }\end{array}$ & Rubiaceae & 5179 & $\begin{array}{l}\text { Dikambali, } \\
\text { Karinga }\end{array}$ & Winter & Fruits & Fruits eaten raw & Jaundice, stomach problems \\
\hline 54. & $\begin{array}{l}\text { Aegle } \\
\text { marmelos } \\
\text { Corr. }\end{array}$ & Rutaceae & 5138 & $\begin{array}{l}\text { Billa, } \\
\text { Bilvapatre, Bel }\end{array}$ & Summer & $\begin{array}{l}\text { Leaves, Fruit } \\
\text { pulp }\end{array}$ & $\begin{array}{l}1-2 \text { Leaves chewed as } \\
\text { raw in early morning. } \\
\text { Fruit pulp eaten raw }\end{array}$ & $\begin{array}{l}\text { Fever, acidity, digestive, } \\
\text { astringent } \\
\text { Male sterility, digestive }\end{array}$ \\
\hline 55. & $\begin{array}{l}\text { Limonia } \\
\text { acidissima } \mathrm{L} .\end{array}$ & Rutaceae & 5189 & $\begin{array}{l}\text { Kaute kai, } \\
\text { Yelka, Naibela }\end{array}$ & Rainy & $\begin{array}{l}\text { Fruit pulp, } \\
\text { Leaves }\end{array}$ & $\begin{array}{l}\text { Fruit pulp grinded to } \\
\text { make chutney, eaten } 2 \text { - } \\
3 \mathrm{~d} \\
\text { Eat equal amount of } \\
\text { fruit pulp with sugar } \\
\text { Take leaf extract orally }\end{array}$ & $\begin{array}{l}\text { All kinds of cough } \\
\text { Pitta, stomach ache } \\
\text { Strengthen immunity }\end{array}$ \\
\hline 56. & $\begin{array}{l}\text { Madhuca } \\
\text { longifolia } \\
\text { (Koenig) } \\
\text { Macbride. }\end{array}$ & Sapotaceae & 5190 & $\begin{array}{l}\text { Ippe gida, } \\
\text { Mahua }\end{array}$ & Summer & Root & $\begin{array}{l}\text { Take outer layer of } \\
\text { root milk by stone and } \\
\text { applied on tonsils }\end{array}$ & Tonsils \\
\hline 57. & $\begin{array}{l}\text { Bacopa } \\
\text { monnieri (L.) } \\
\text { Wettst. }\end{array}$ & $\begin{array}{l}\text { Scrophulariace } \\
\text { ae }\end{array}$ & 5148 & $\begin{array}{l}\text { Neeru bhrahmi, } \\
\text { Timare }\end{array}$ & Rainy & Leaves & $\begin{array}{l}\text { Drink two spoon of } \\
\text { leaves extract at early } \\
\text { in the morning with } \\
\text { empty stomach }\end{array}$ & $\begin{array}{l}\text { Nerve tonic, Strengthen } \\
\text { immunity }\end{array}$ \\
\hline 58. & $\begin{array}{l}\text { Balanetes } \\
\text { roxburghii } \\
\text { Planch. }\end{array}$ & Simarubaceae & 5149 & $\begin{array}{l}\text { Ingalkyan kai, } \\
\text { Hingin kai }\end{array}$ & Summer & $\begin{array}{l}\text { Fruit pulp, } \\
\text { Fruits }\end{array}$ & $\begin{array}{l}\text { Dried fruit pulp mixed } \\
\text { in Jaggary to make a } \\
\text { tablets and take for } 2-3 \\
\text { d with empty stomach } \\
\text { Fruit epicarp paste } \\
\text { applied on eyes }\end{array}$ & $\begin{array}{l}\text { Jaundice, stomach problems, } \\
\text { purgative } \\
\text { Eye diseases, skin problems }\end{array}$ \\
\hline 59. & $\begin{array}{l}\text { Physalis } \\
\text { minima L. }\end{array}$ & Solanaceae & 5203 & $\begin{array}{l}\text { Gudde hannu, } \\
\text { Sannaguppate } \\
\text { gida }\end{array}$ & Winter & Leaves & $\begin{array}{l}\text { Leaf extract applied on } \\
\text { leg or any swollen part } \\
\text { of the body }\end{array}$ & Edima \\
\hline 60. & $\begin{array}{l}\text { Solanum } \\
\text { nigrum L. }\end{array}$ & Solanaceae & 5211 & $\begin{array}{l}\text { Kaamini gida, } \\
\text { Ganike palya }\end{array}$ & $\begin{array}{l}\text { Throughou } \\
\mathrm{t} \text { the year }\end{array}$ & Leaves & $\begin{array}{l}\text { Drink leaf extract 3-4 d } \\
\text { (Precautions: eat only } \\
\text { Sorghum rotti/bread } \\
\text { and Brinjal curry). } \\
\text { Leaves boiled or burn } \\
\text { and eaten }\end{array}$ & $\begin{array}{l}\text { Jaundice, stomach problems } \\
\text { Pitta, mouth ulcer }\end{array}$ \\
\hline 61. & Lantana & Verbenaceae & 5187 & Kaagi gida, & Throughou & Leaves, & Leaves paste mixed & Skin diseases \\
\hline
\end{tabular}




\begin{tabular}{|c|c|c|c|c|c|c|c|c|}
\hline S. No. & $\begin{array}{l}\text { Botanical } \\
\text { name }\end{array}$ & $\begin{array}{l}\text { Family/Subfa } \\
\text { mily }\end{array}$ & $\begin{array}{l}\text { HGUG } \\
\text { voucher } \\
\text { no. }\end{array}$ & $\begin{array}{l}\text { Bidar local } \\
\text { name } / \mathrm{s}\end{array}$ & $\begin{array}{l}\text { Available } \\
\text { season }\end{array}$ & Part/s used & Preparation/dosage & Ailment/s or disease/s \\
\hline & camara $\mathrm{L}$. & & & $\begin{array}{l}\text { Gangoda, } \\
\text { Shisha, } \\
\text { Kammari }\end{array}$ & $t$ the year & Fruits & $\begin{array}{l}\text { with lime powder and } \\
\text { applied on the face } \\
\text { Ripped fruit eaten raw }\end{array}$ & Loose motion \\
\hline 62. & $\begin{array}{l}\text { Cissus } \\
\text { quadrangulari } \\
s \mathrm{~L} .\end{array}$ & Vitaceae & 5162 & Sandu balli & Rainy & Stem & $\begin{array}{l}\text { Stem paste mixed in } \\
\text { butter and applied on } \\
\text { bone fracture or } \\
\text { fingers }\end{array}$ & Bone fracture \\
\hline 63. & $\begin{array}{l}\text { Tribulus } \\
\text { terrestris L. }\end{array}$ & Zygophyllaceae & 5217 & Neggi mullu & $\begin{array}{l}\text { Throughou } \\
t \text { the year }\end{array}$ & $\begin{array}{l}\text { Fruits, } \\
\text { Whole plant }\end{array}$ & $\begin{array}{l}\text { Fruit powder of } \\
\text { Tribulus terrestris and } \\
\text { root powder of } \\
\text { Achyranthus aspera } \\
\text { mixed in water and } \\
\text { drink daily } 2 \text { times. } \\
\text { Whole plant ash mix in } \\
\text { water } \\
\text { Then powder will } \\
\text { appears on the surface } \\
\text { of water than the } \\
\text { powder is mixed in } \\
\text { honey and eaten. }\end{array}$ & $\begin{array}{l}\text { Kidney stone, stomach ache, } \\
\text { excretory disorders } \\
\text { Asthma, bronchitis }\end{array}$ \\
\hline
\end{tabular}

\section{CONCLUSION}

The above investigation deals with 63 edible wild plant species used as medicine for various diseases in Bidar district. The information was gathered from different 42 people of local medicinal practitioners in the study area. Day by day the, people will be attracting towards the herbal medicine for their no side effect. There is less information about wild edible plants with their uses in the district. So far, it is important to document and evaluate this knowledge for their medicinal value of plants. The present paper is communicated to gives more and more medicinal properties of edible wild plants. It is desirable to use of proper plants for particular diseases by their correct formulations without any confusion.

\section{ACKNOWLEDGEMENT}

Author is very grateful to all informants of Bidar district for their willingness sharing knowledge regard wild edible plants used as medicine for various diseases.

\section{FUNDING}

Nil

\section{AUTHORS CONTRIBUTIONS}

All the authors have contributed equally.

\section{CONFLICT OF INTERESTS}

Author herewith declares that there is no any conflict of interest.

\section{REFERENCES}

1. Jain SK. Some less known plant foods among the tribal of Purulia district, West Bengal. Sci Cult. 1964;30:285.

2. Bagul RM. Some ethnomedicinal plant species of satpuda forest region of east Khandesh Jalgaon district, Maharashtra. J New Biol Rep. 2013;2(3):264-71.

3. WHO (World Health Organization). Quality Control for Medicinal plant material. New Delhi: AITBS Publishers; 1998. p. 46.

4. Oak Gayatri, Kurve Poonam, Kurve Siddhisha, Pejaver Madhuri. Ethnobotanical studies of edible plants used by tribal women of Thane district. J Med Plants Stud. 2015;3(2):90-4.

5. Champion GH, Seth SK. A revised survey of the forest types of India. New Delhi: Government of India; 1968.

6. Roy Subarna, Hegde Harsha V, Bhattacharya Debdutta, Upadhya Vinayak, Kholkute Sanjiva D. Tribes in Karnataka: status of health research. Indian J Med Res. 2015;141(5):67387. doi: 10.4103/0971-5916.159586, PMID 26139788.

7. Venkataraman K. India's biodiversity act. 2002 and its role in conservation. Vol. 50. International Society for Tropical Ecology; 2009. p. 23-30.

8. Gamble JS, Fisher CEC. Flora of the presidency of madras. Reprinted edition: Vol. IIII. Calcutta: BSI; 1957.
9. Saldanha CJ. Flora of Karnataka. Oxford and IBH: publishing Co. New Delhi; 1984.

10. Singh NP. Flora of eastern Karnataka. Reprinted edition, Vol. III: Mittal publications Delhi; 1988.

11. Seetharam YN, Kotresh K, Uplaonkar SB. Flora of Gulbarga District. Gulbarga, Karnataka: Gulbarga University; 2000. p. 160.

12. Amit Tomar. Folk medicinal uses of plant roots from Meerut district, Uttar Pradesh. Indian J Trad Knowl. 2009;8:298-301.

13. Mandal Animesh, Bishayee Anupam. Trianthema portulacastrum Linn. displays anti-inflammatory responses during chemically induced rat mammary tumorigenesis through simultaneous and differential regulation of NF- $\kappa \mathrm{B}$ and Nrf2 signaling pathways. Int J Mol Sci. 2015;16(2):2426-45. doi: 10.3390/ijms16022426, PMID 25622256.

14. Yugandhar P, Savithramma N, Suhrulatha D. Traditional medicinal plants used by local people of kailaskona-a sacred grove of chittoor District, Andhra Pradesh, India. 2015;7:407-11.

15. Archana Singh. An ethnobotanical study of medicinal plants in sonebhadra district of Uttar, Pradesh, India with reference to their infection by foliar fungi. J Med Plants Res. 2012;6(14):2727-46. doi: 10.5897/JMPR10.895.

16. Rajasab AH, Isaq $M$. Documentation of folk knowledge on edible wild plants of North Karnataka. Indian J Trad Knowl. 2004;3:419-29.

17. Balemie Kebu, Kebebew Fassil. Ethnobotanical study of wild edible plants in Derashe and Kucha Districts, South Ethiopia. J Ethnobiol Ethnomed. 2006;2:53. doi: 10.1186/1746-4269-253, PMID 17184523.

18. Reddy KN, Chiranjibi Pattanaik Reddy CS, Raju VS. Traditional knowledge on wild food plants in Andhra Pradesh. Indian J Trad Knowl. 2007;6:223-9.

19. Pegu Rajeswar, Gogoi Jitu, Tamuli Ajit K, Teron Robindra. eth no botanical study of wild edible plants in poba reserved Forest, Assam, India: multiple functions and implications for conservation. Res J Agric For Sci. 2013;1:1-10.

20. Shiddamallayya N, Prashant Kumar GM. Documentation of wild leafy vegetables of Hassan district, Karnataka. Int J Pure Appl Biosci. 2014;2:202-8.

21. Nadkarni KM. Indian material medica. 3rd ed. Bombay: Bombay Popular Prakashan; 1954. p. 690.

22. Rameshkumar A, Sivasudha T, Jeyadevi R, Sangeetha B, Smilin bell Aseervatham G, Maheshwari M. Profiling of phenolic compounds using UPLC-QTOF-MS/MS and nephroprotective activity of Indian green leafy vegetable Merremia emarginata (Burm. f). Food Res Int. 2012. p. 1016.

23. Rameshkumar A, Sivasudha T, Jeyadevi R, Sangeetha B, Arul Ananth D, Smilin Bell Aseervatham G, Nagarajan N, Renganathan $\mathrm{R}$, Kathiravan A. In vitro antioxidant and antimicrobial activities of Merremia emarginata using thioglycolic acid-capped cadmium telluride quantum dots. Colloids and Surfaces B. Biointerfaces. 2013;101:74-82. 
24. Purushoth Prabhu T, Shine Sudhev, Clement Atlee W, Suresh R, Balasubramanian S. Pharmacognostic studies on whole plant of Merremia emarginata burm. F (Convolvulaceae). Int J Pharmacognosy and Phytochemical Research. 2004;6:1002-6.

25. Devadasu Ch, Naga Jyothi N, Srinivasa Babu P, Afzal Basha SK. Biological evaluation of merremia emarginata for antibacterial, antifungal and antioxidant activities. World J Pharm Pharm Sci. 2017;6:1509-25.

26. Shivanna MB, Mangala KR, Mahishi Parinitha. Ethno-medicinal knowledge of Lambani community in Chikmagalur district of Karnataka, India. J Med Aromat Plants Sci. 2008;30:105-8.

27. Kuru Suresh Kottaimuthu R, Selvin Jebaraj Norman T, Kumuthakalavalli R, Sabu M Simon. Ethnobotanical study of medicinal plants used by malayali tribals in Kollihills of Tamil nadu, India. IJRAP. 2011;2:502-8.
28. Hazarika TK, Lalramchuana Nautiyal BP. Studies on wild edible fruits of Mizoram, India used as ethnomedicine. Genetic Resources and Crop Evolution-An International Journal 2012;59:1767-76.

29. Anu Shrivastava, Shikha Roy. Cucurbitaceae: a ethno medicinally Important vegetable family. J Med Plants Stud. 2013;1:16-20.

30. Upinder Gill S, Uppalapati Srinivasa R, Jin Nakashima, Kirankumar S Mysore. Characterization of Brachypodium distachyon as a non-host model against switchgrass rust pathogen Puccinia emaculata. BMC Plant Biol. 2015;15:113.

31. Mahadkar Shivprasad, Rane Megha, Satavi Vaibhav. Documentation and ethnobotanical survey of wild edible plants from Palghar district. Asian J Pharm Clin Res. 2016;9:16-9. 